\title{
Experimental characterization of a new class of polymeric-wire coiled transducers
}

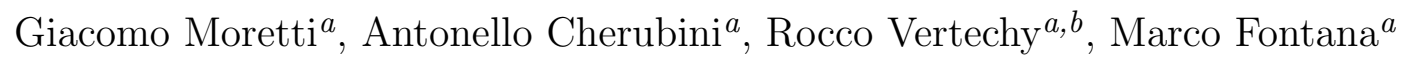 \\ ${ }^{a}$ PERCRO-SEES Laboratory, Scuola Superiore Sant'Anna, Pisa, Italy \\ ${ }^{b}$ Department of Industrial Engineering, University of Bologna, Italy
}

\begin{abstract}
The recent discovery of a new kind of thermo-active coiled polymeric wires has opened new perspectives for the implementation of a novel class of actuators that can be easily and effectively manufactured using low-cost materials such as sewing threads or fishing lines. These new devices feature large displacements in response to temperature variations and show very promising performance in terms of energy and power densities.

With the aim of providing information and data useful for the future engineering applications of polymeric coiled actuators, a custom experimental test-bench and procedure have been developed and employed to characterise their thermo-mechanical response. Such a test-bench has been designed to run isothermal and isometric tensile tests on a set of sample actuators that are fabricated with a repeatable process.

This paper provides technical details on the manufacturing process of such sample actuators and on the design and operation of the test-bench. Preliminary experimental results are finally reported.
\end{abstract}

Keywords: Nylon, Coil, Actuators, Springs, Thermo, Mechanical, Characterization, Experimental, TMA

\section{INTRODUCTION}

In the field of thermal actuators, the recently discovered of polymeric-wire coils represents very a promising new technology. ${ }^{1}$ In fact, cheap highly-drawn polymeric wires can be easily turned into powerful and reliable actuators that can shrink or extend when exposed to a temperature increase.

Haines et al. ${ }^{1}$ investigated a variety of materials, e.g. nylon-6, nylon- 6.6 and polyethylene, building several coiled actuators with different geometries, and testing them in different embodiments (e.g, embedded in braids which change porosity in response to temperature, or employed as artificial muscles to lift loads). In these investigations, nylon exhibited very good performance, showing actuation energy densities up to $2.6 \mathrm{~kJ} / \mathrm{kg}$, low hysteresis, and repeatable behaviour. Thanks to these features, the economic potential of nylon-wire coiled actuators is very high.

In order to provide a complete description of the behaviour of these actuators, a thermo-mechanical characterization is required. This article describes an experimental setup that has been particularly developed for this purpose.

Attention is focused on homochiral ${ }^{1}$ actuators manufactured by autocoiling, ${ }^{2}$ that are obtained from a monofilament nylon fibre, hereafter referred to as Nylon Coil (NC).

The paper is structured as follows. Section 2 describes a manufacturing process of NCs that has been employed for the preparation of the specimens. Section 3 provides an overview of a preliminary explanation of their working principle. Section 4 presents the dedicated test-bench which has been designed for the characterization of NCs. Finally, some preliminary results are shown in Section 5.

Further author information: (Send correspondence to Marco Fontana) E-mail: m.fontana@sssup.it, Telephone: +39050882516

Behavior and Mechanics of Multifunctional Materials and Composites 2015, edited by Nakhiah C. Goulbourne Proc. of SPIE Vol. 9432, 94320P - @ 2015 SPIE · CCC code: 0277-786X/15/\$18 · doi: 10.1117/12.2084338 


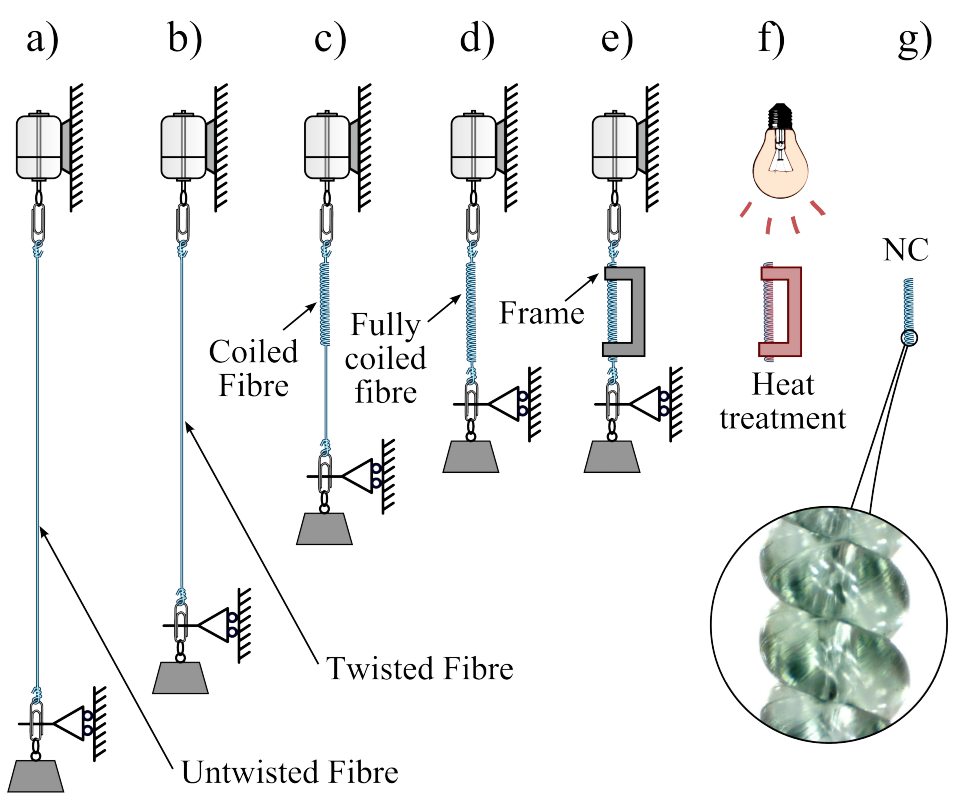

Figure 1. NCs manufacturing by autocoiling. - a) The precursor fibre has the top end connected to the output of a motor, and the bottom end rigidly attached to a weight which is prevented from rotation and is free to slide up and down. b) As the motor starts rotating, the fibre twists. c) Coiling is generated by mechanical instability (autocoiling). d) When the whole length is coiled, the motor stops rotating. e) A rigid frame is attached to the coil to prevent it from untwisting and losing its shape. f) The coil is subjected to heat treatment. g) The coil can be removed from the rigid frame, keeping its shape, and it is ready to be employed as thermal actuator.

\section{MANUFACTURING BY AUTOCOILING}

This section presents the 'autocoiling' process of NCs that has been employed for the manufacturing of sampleactuators.

A simple nylon wire (hereafter referred to as 'precursor fibre') is held vertically with a constant tension provided by an attached weight (Fig. 1.a), the top end is rotated around the longitudinal axis of the fibre at a constant altitude and the bottom end can freely slide upwards without rotating. While the top end is rotated, the fibre twists and shortens (Fig. 1.b), then it naturally starts to deform assuming a helical shape (Fig. 1.c), until it is fully coiled and the rotation is stopped (Fig. 1.d). After the coiling process, a heat-treatment-frame (Fig. 2) is fixed onto the NC (Fig. 1.e) and the manufacturing weight is then removed. Thanks to the heattreatment-frame, the NC coil does not lose twist and can be heat-treated with its shape fully coiled (Fig. 1.f). Finally, after heat treatment, the $\mathrm{NC}$ can be removed from the heat-treatment-frame (Fig. 1.g). At this point, the NC maintains its shape and is ready to be employed as a thermal actuator.

With this procedure, the chirality of the twist given to te precursor fibre and that of the coil are the same. The coil is then called 'homochiral'. Heterochiral coils (with coil chirality opposite to that of the twist of the precursor fibre) may be obtained with a different procedure, called 'mandrel coiling', wrapping the twisted non-coiled fibres around a mandrel. ${ }^{1}$

\section{ACTUATION PRINCIPLE}

Homochiral NCs shorten when heated. This contraction is caused mainly by the radial thermal expansion of the precursor fibre, together with the axial thermal shrinkage in the precursor fibre. Nylon is especially suited for this application because, when heated, it has a positive radial expansion (approximately $5 \%$ ) and a negative axial expansion (approximately $-2 \%$ ). The working principle has been explained by Mirvakili et al. ${ }^{3}$ and is resumed in the following. 

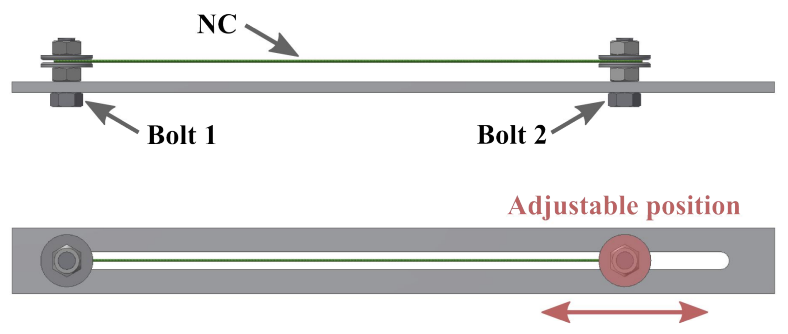

Figure 2. Heat-treatment-frame - After coiling, the heat-treatment-frame is fastened to the NC (Fig. 1.e). Then the manufacturing weight can be removed and the NC can be easily handled for heat treatment.

The precursor fibre before the manufacturing can be thought as a long and thin cylinder made of a bundle of polymeric chains. Imagine to twist the fibre (without coiling) as in Fig. 1.b. In this twisted configuration, polymeric chains have helical shape, as shown in Fig. 3.a. In Fig. 3.a, with reference to one helical pitch length, the right triangle obtained by unrolling the polymeric chain is shown. For such a triangle, the following equation applies:

$$
\lambda^{2}=l^{2}+\left(\pi d_{\mathrm{t}} n\right)^{2}
$$

where $n$ is the number of twist turns, $\lambda$ is the untwisted fibre length, $l$ is the twisted fibre length and $d_{\mathrm{t}}$ is the twisted fibre diameter. By differentiating Eq. 1, and using trigonometric definitions, the following relationship is obtained:

$$
\frac{\mathrm{d} n}{n}=\frac{1}{\sin ^{2} \alpha_{\mathrm{f}}} \frac{\mathrm{d} \lambda}{\lambda}-\frac{1}{\tan ^{2} \alpha_{\mathrm{f}}} \frac{\mathrm{d} l}{l}-\frac{\mathrm{d} d_{\mathrm{t}}}{d_{\mathrm{t}}}
$$

where $\alpha_{\mathrm{f}}$ is the fibre bias angle (Fig. 3.a), which equals zero when the fibre is not twisted (i.e. $n=0$ ).

It has been previously observed that, in presence of temperature variations, $\mathrm{d} l / l$ is small compared to the other addenda, ${ }^{1}$ therefore from Eq. 2 it is possible to see that a decrease in $\lambda$ or an increase in $d_{\mathrm{t}}$ both lead to a decrease in the fibre twist $n$. This explanation, that was done for the outer fibres of the cylinder, holds true for any inner layer of the cylinder. Nylon fibres, which features positive variations in $\lambda$ and negative variations in $d_{\mathrm{t}}$ when heated, provide untwist when their temperature increases.

It can be now demonstrated that a decrease in twist generates a decrease in coil length.

We assume that, even when the fibre is fully coiled, it untwists when heated.

The central axis of a coiled fibre with mean diameter $D$ and pitch $p$ (Fig. 3.b) can be modelled as a circular helix having parametric coordinates:

$$
\vec{r}=\left(\begin{array}{c}
x \\
y \\
z
\end{array}\right)=\left(\begin{array}{c}
(D / 2) \cos \theta \\
(D / 2) \sin \theta \\
p \theta /(2 \pi)
\end{array}\right)
$$

where $\theta$ is the azimuth angle of the cylindrical coordinates parametrization.

Thanks to the geometrical properties of the circular helix it is possible to derive a convenient kinematic relation between twist variations of the fibre and coil length variations. More specifically, it is possible to compute the torsion curvature, $\tau$, by parametrizing the helix formed by the central axis with the arc length $s$ and using the tangent, normal and binormal unit vectors, $\vec{T}, \vec{N}$, and $\vec{B}$ of Frenet-Serret reference frame: ${ }^{3}$

$$
\vec{T}=\frac{\mathrm{d} \vec{r}}{\mathrm{~d} s} \quad \vec{N}=\frac{\frac{\mathrm{d} \vec{T}}{\mathrm{~d} s}}{\left|\frac{\mathrm{d} \vec{T}}{\mathrm{~d} s}\right|} \quad \vec{B}=\vec{T} \times \vec{N}
$$




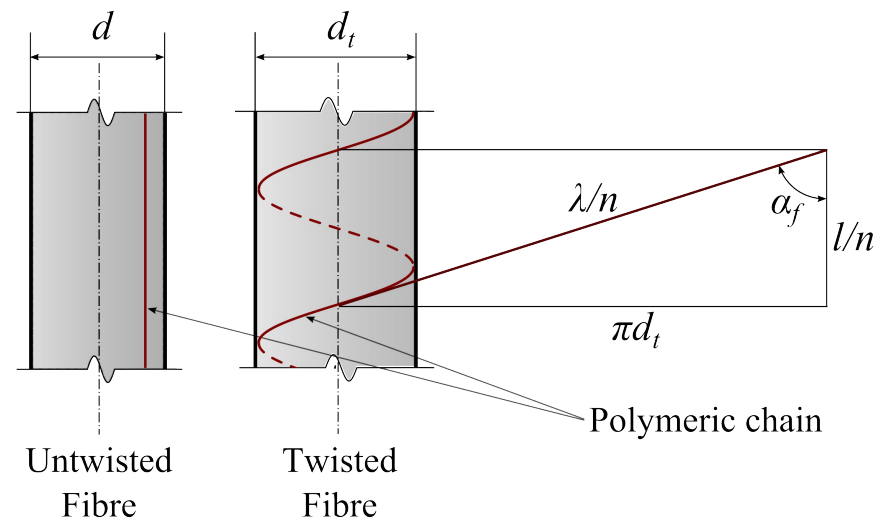

(a)

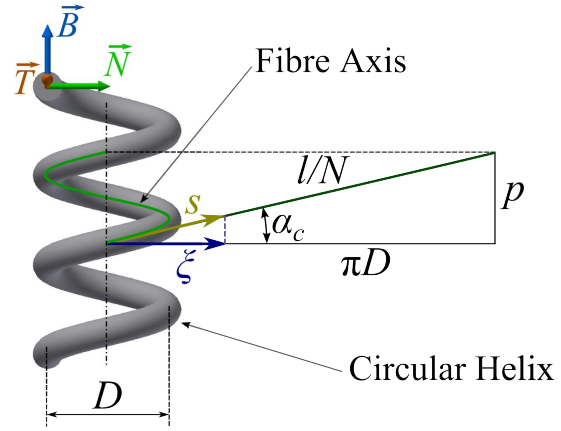

(b)

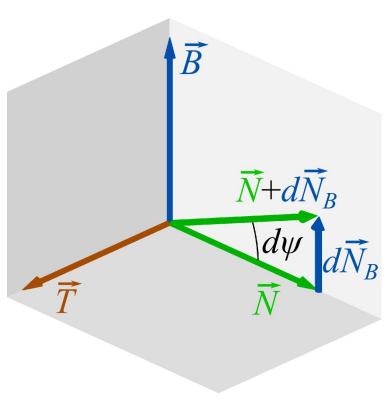

(c)

Figure 3. Nylon coil working principle - a) Uncoiled fibre before and after twisting, b) circular helix used to model a coiled fibre, c) infinitesimal rotation $\mathrm{d} \psi$ of the normal unit vector around the tangent axis, $\vec{T}$. Here, $\mathrm{d} N_{B}$ represents the projection along $\vec{B}$ of the infinitesimal variation of $\vec{N}$

$$
\begin{array}{lr}
s=\sqrt{\xi^{2}+z^{2}} & \xi=\frac{D}{2} \theta \\
\tau=\frac{\mathrm{d} \vec{N}}{\mathrm{~d} s} \cdot \vec{B}=\frac{\mathrm{d} \vec{N}_{\mathrm{B}}}{\mathrm{d} s} & \mathrm{~d} s=\frac{1}{2 \pi} \sqrt{\pi^{2} D^{2}+p^{2}} \mathrm{~d} \theta \\
N \pi D=l \cos \left(\alpha_{\mathrm{c}}\right) & L=l \sin \left(\alpha_{\mathrm{c}}\right)
\end{array}
$$

where $\xi$ is the circular coordinate defined in Fig. 3.b and $z$ is defined in Eq. (3); $L$ is the coil length; $\alpha_{\mathrm{c}}$ is the coil bias angle defined as the angle of the fibre with respect to the plane normal to the coil axis; $N$ is the number of coils of the actuator. The pitch of the helix reads as $p=L / N$.

The torsion $\tau$ measures how much the curve deviates from the osculating plane and defines the local rotation, $\psi$, of the normal vector around the tangent axis per unit fibre length ${ }^{4}$ as shown in Fig. 3.c, thus resulting in $\tau=\mathrm{d} \psi / \mathrm{d} s$. 
Since torsion $\tau$ for a circular helix is constant along $s$, torsion variations induced by the coil deformation result in twist variations.

Now, suppose to deform the coil from a configuration 1 to a configuration 2, respectively with coil length of $L_{1}$ and $L_{2}$, coil diameter $D_{1}$ and $D_{2}$, bias angle $\alpha_{1}$ and $\alpha_{2}$ and number of twists $n_{1}$ and $n_{2}$.

In these configurations, the following equations apply:

$$
\begin{array}{ll}
N \pi D_{1}=l \cos \left(\alpha_{\mathrm{c} 1}\right) & L_{1}=l \sin \left(\alpha_{\mathrm{c} 1}\right) \\
N \pi D_{2}=l \cos \left(\alpha_{\mathrm{c} 2}\right) & L_{2}=l \sin \left(\alpha_{\mathrm{c} 2}\right),
\end{array}
$$

where variations in twisted fibre length, $l$, were again neglected.

Using Eqs. 3-7, the resulting difference in the number of twists per unit of fibre length is:

$$
\frac{\Delta n}{l}=\frac{n_{2}-n_{1}}{l}=\frac{\Delta \tau}{2 \pi l}=\frac{\sin \left(\alpha_{\mathrm{c} 2}\right) \cos \left(\alpha_{\mathrm{c} 2}\right)}{\pi D_{2}}-\frac{\sin \left(\alpha_{\mathrm{c} 1}\right) \cos \left(\alpha_{\mathrm{c} 1}\right)}{\pi D_{1}}
$$

which can be rewritten as:

$$
\Delta L=L_{2}-L_{1}=\Delta n \frac{l}{N}
$$

thus demonstrating that a decrease in fibre twists $n$ kinematically corresponds to a decrease in coil length $L$.

To summarise, in an homochiral NC, an increase in temperature generates two effects: an increase in fibre radius and a decrease in fibre length. Both these two effects lead to a fibre untwist. The latter finally generates a shortening in coil length.

\section{TEST-BENCH}

This section describes a test-bench that has been specifically built for performing a Thermo-Mechanical Analysis (TMA) of NCs: elongation, load and temperature are measured.

\subsection{Temperature Chamber}

NCs must be tested at high strain over a wide range of temperatures (up to $200^{\circ} \mathrm{C}$ ) with the possibility of visual inspection and special clamping to securely hold them in place. In order to meet this set of requirements, a dedicated tensile testing machine equipped with a custom temperature chamber has been designed and built. The system, shown in Fig. 4, consists of a glass-walled chamber of variable length that houses a nylon coil specimen which is firmly held by two clamps. The chamber is composed by two coaxial glass tubes, an upper one that is fixed, and a lower one that slides up and down. The position of the lower tube is controlled with a linear actuator, thus allowing to know the elongation of the $\mathrm{NC}$, the load is measured with a load cell thermally insulated from the glass chamber. The chamber temperature is set by means of an air flow at controlled temperature. As shown in the left photograph of Fig. 4, hot air enters the chamber from a pipe located on top of the upper tube and exits through the gap between the tubes. The temperature of the chamber is measured with two thermocouples located near the top and bottom ends of the NC.

The coaxial-glass-tubes layout ensures sufficiently uniform heating throughout the chamber and allows an easy mounting procedure for the specimen.

The materials used to build the test machine are metals, wood and glass. The specimen can reach temperatures up to $200^{\circ} \mathrm{C}$ while the load cell is kept very close to the ambient temperature thanks to the thermal insulation assembly. The latter is made by four wooden layers with an air chamber in between. 


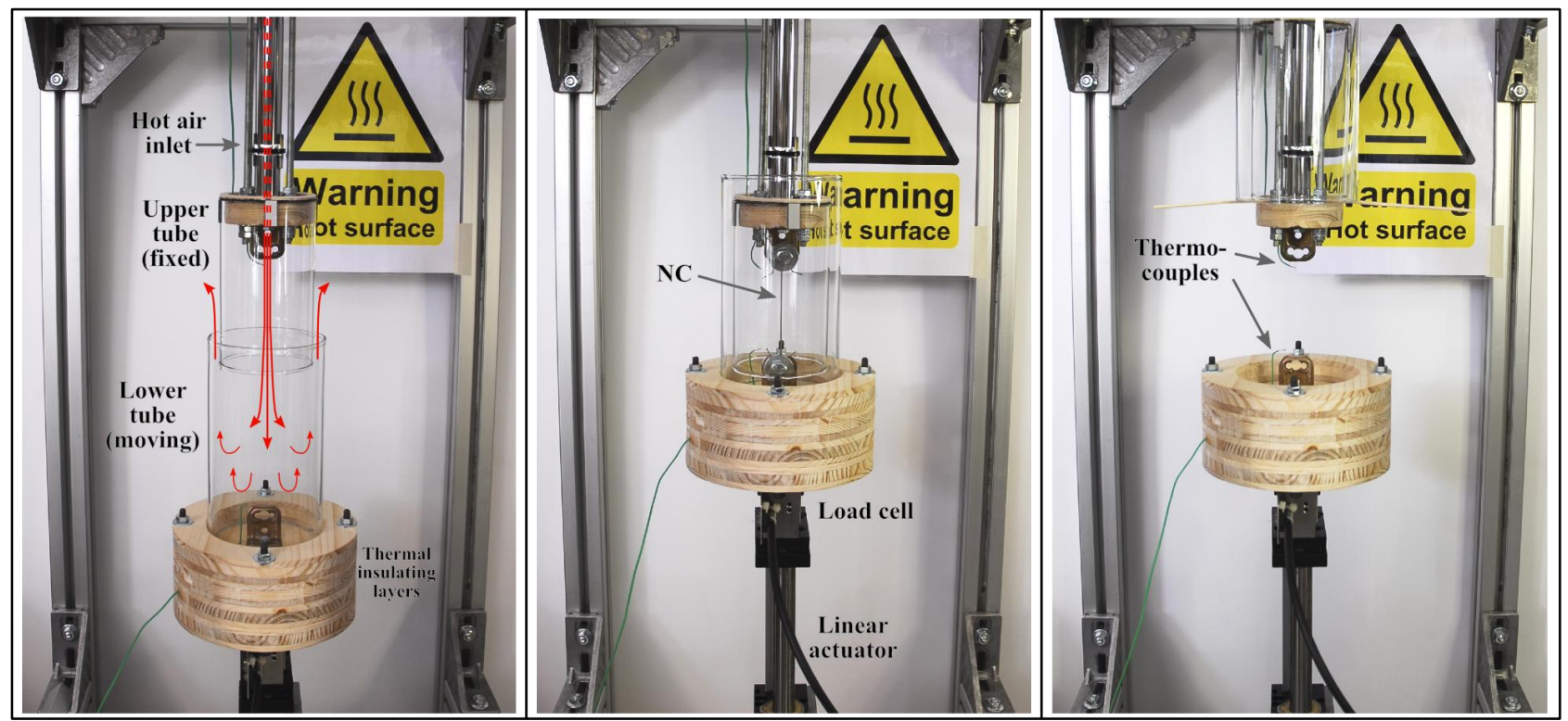

Figure 4. Test-bench for nylon coils - The test-bench fully elongated (left), at zero position (centre), and with lifted tubes for easy specimen mounting (right).

\subsection{Multi-Washer Clamp}

The development of a reliable clamp able to keep the actuator in place during its operation was a critical task that required several trial-and-error iterations. The clamp must be tight enough to withstand the relatively high loads in the fibre and the high temperatures, but at the same time, it should not damage the coil by holding it too tight. Finally it has to ensure a reliable and, above all, repeatable clamping force. The solution proposed here satisfies all these requirements.

Several kinds of clamps have been tried and the one that has proven to be the most reliable and easy to mount is here referred to as Multi-Washer Clamp (MWC), which is shown in Fig. 5.

With reference to Fig. 5, the three steel washers ensure the proper distribution of contact pressure. The VHB layer has the important functions of, first, holding the nylon coil in place while nut A is tightened and, second, ensuring enough friction between the nylon coil and the steel washers during actuation so that the NC is prevented from sliding. The sticky VHB layer can be covered by a non-adhesive layer (e.g a paper layer) on both sides for better handling. In case the VHB layer has the paper coating, the central washer is needed. On the contrary, if the VHB is directly glued to one of the outer washers, then the central washer is not mandatory. The spring-steel spacer wire allows to fasten nut A with a repeatable axial force without cutting the nylon coil. The nylon coil and the spacer clip should not overlap when clamping. After having fastened nut A, the clamped nylon coil can be fixed to the test-bench by using nut B.

The MWC has proven to be reliable at a maximum applied tensile load of 1) $80 \mathrm{MPa}$ of nominal stress* up to $\left.150^{\circ} \mathrm{C}, 2\right) 120 \mathrm{MPa}$ (nominal breaking stress) at ambient temperature. In these conditions, a NC with fibre diameter $d=0.5 \mathrm{~mm}$ and outer diameter $D=1.2 \mathrm{~mm}$ was clamped with the following mechanical components: bolt M8, outer washers $8 \times 24 \mathrm{~mm}$, central washer $12 \times 24 \mathrm{~mm}, 8 \times 24 \times 0.5 \mathrm{~mm}$ VHB layer and $0.8 \mathrm{~mm}$ spacer wire.

\subsection{Actuators, sensors and electronics}

A brushless linear motor P01-37x120F/200x280-HP by LinMot is used to actuate the test-bench. The elongation is measured with a repeatability of $\pm 0.05 \mathrm{~mm}$ thanks to the embedded encoder of the motor.

The load is measured by the load cell DS Europe 535QD A1 with a maximum error of $0.06 \mathrm{~N}$.

\footnotetext{
${ }^{*}$ The nominal stress is computed as the ratio between the applied load and the untwisted fibre section.
} 


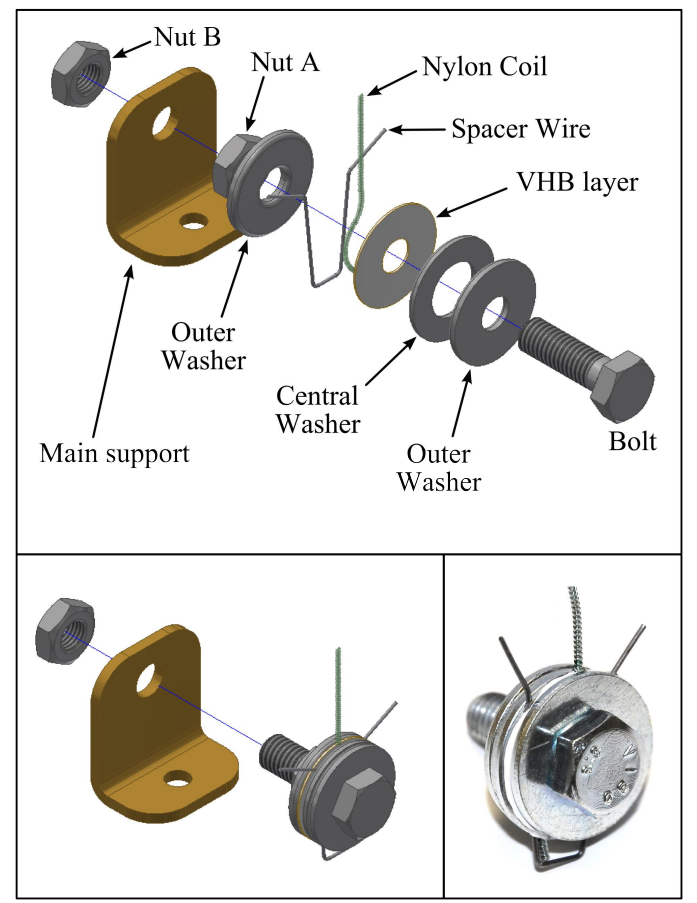

Figure 5. Multi-Washer Clamp - Detailed CAD view (top). CAD model and photograph of a clamped nylon coil (bottom).

Two type-K thermocouples are placed inside the chamber to monitor the even distribution of temperature. The temperature is measured with a total error of $\pm 5^{\circ} \mathrm{C}$.

A real-time machine (Performance real-time target machine by SpeedGoat) running the MatLab xPC Target software environment is employed to control NC elongation and to measure load and temperature.

\section{TESTS AND PRELIMINARY RESULTS}

In order to obtain the thermo-mechanical characterization of NCs, the coaxial-tubes test-bench can be used to perform several kinds of tests on NCs, for example:

- Isothermal tests: the temperature is kept constant, the NC is cyclically stretched and the load is measured.

- Isometric tests: the $\mathrm{NC}$ is kept at a constant deformation, the temperature is changed and the load is measured.

A Reference Coil (RC) has been used during the tests. The $\mathrm{RC}$ was produced from a $0.5 \mathrm{~mm}$ nylon-6 monofilament fishing line by Teklon Carson, by autocoiling under $25 \mathrm{MPa}$ of constant nominal stress during manufacturing, with in-air heat treatment at $150^{\circ} \mathrm{C}$ for 1 hour. During isothermal tests, the RC undergoes cyclic strain starting form an undeformed length at ambient temperature, $L_{0}$, equal to $70 \mathrm{~mm}$. The maximum elongation, $\Delta L$, is $35 \mathrm{~mm}$, and the deformation speed is $2 \mathrm{~mm} / \mathrm{s}$. During isometric tests, the RC is stretched, $\Delta L$ is fixed and the temperature is cyclically increased and decreased.

An example of stress-strain curves during isothermal tests at two different temperatures is shown in Fig. 6. The graph shows 10 elongation cycles on the $\mathrm{RC}$ at $30^{\circ} \mathrm{C}$ and $120^{\circ} \mathrm{C}$. After a first 'training' cycle the elongation path becomes highly repeatable. Hysteresis can be calculated on the average the last two cycles and expressed as the amount of dissipated energy over the elastic energy provided to the sample during the stretching phase. Isothermal tests show a decrease in hysteresis with increasing temperatures, ranging from $33 \%$ to $11 \%$ at $30^{\circ} \mathrm{C}$ and $120^{\circ} \mathrm{C}$, respectively. This hysteresis reduction with rising temperature is a known phenomenon in polymers 


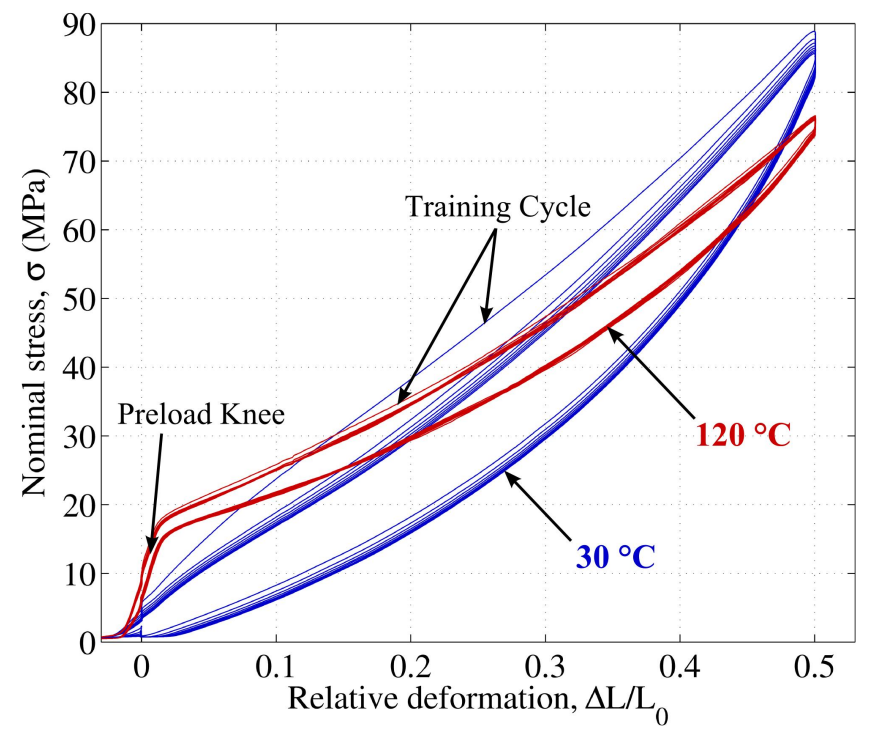

Figure 6. Example of isothermal test results - The pictures shows 10 elongation cycles at two different temperatures. Hysteresis is visibly lower at higher temperatures.

science and has been observed on other materials, like rubber. ${ }^{5}$

The first cycle for both temperatures is referred to as 'training cycle': after this cycle the response of the specimen becomes repeatable. At $120^{\circ} \mathrm{C}$ and at very low $\Delta L$, adjacent coils touch each other because of thermal shrinkage thus giving the 'preload knee' shape shown in the Figure. As for the energy density, the RC showed densities up to $0.8 \mathrm{~kJ} / \mathrm{kg}$ during thermal actuation and up to $3.5 \mathrm{~kJ} / \mathrm{kg}$ if they were simply employed as springs in isothermal conditions (safely far from the breaking point). ${ }^{2}$ These are very interesting values that may be improved by optimizing the design and the testing conditions of the samples and they are of the same orders of magnitude of the energy density of Dielectric Elastomers ${ }^{6}$ and Shape Memory Alloys. ${ }^{7}$

\section{CONCLUSIONS}

This article presents a test-bench specifically designed to characterize polymeric-wire coiled actuators, a new class of thermo-mechanical transducers made of simple polymeric wires, which have been shown to provide very promising actuation performance. The presented test-bench is based on a coaxial-tubes glass temperature chamber and has been built to perform cyclic tensile tests over a wide range of temperatures (up to $200^{\circ} \mathrm{C}$ ). The proposed layout allows to easily mount and to securely clamp the specimens to the machine.

Using the presented setup, a reference coil made of a nylon-6 fishing line has been tested within a temperature range between $30^{\circ} \mathrm{C}$ and $120^{\circ} \mathrm{C}$ in order to obtain a thermo-mechanical characterization of the sample-actuator. Isothermal stress-strain curves have been obtained, hysteresis and actuation energy density have been measured. Experimental results confirm that the technology is very promising in applications requiring lightweight, electromagneto insensitive and low-cost actuators.

\section{ACKNOWLEDGMENTS}

This work was carried out with funds from Scuola Superiore Sant'Anna, Pisa, Italy. 


\section{REFERENCES}

[1] C. S. Haines, M. D. Lima, N. Li, G. M. Spinks, J. Foroughi, J. D. Madden, S. H. Kim, S. Fang, M. J. De Andrade, F. Göktepe, Ö. Göktepe, S. M. Mirvakili, S. Naficy, X. Lepr, J. Oh, M. E. Kozlov, S. J. Kim, X. Xu, B. J. Swedlove, G. G. Wallace, and R. H. Baughman, "Artificial muscles from fishing line and sewing thread," Science 343(6173), pp. 868-872, 2014.

[2] A. Cherubini, G. Moretti, R. Vertechy, and M. Fontana, "Experimental characterization of thermallyactivated artificial muscles based on coiled nylon fishing lines," Smart Materials and Structures., 2015. In Review.

[3] S. M. Mirvakili, A. R. Ravandi, I. W. Hunter, C. S. Haines, N. Li, J. Foroughi, S. Naficy, G. M. Spinks, R. H. Baughman, and J. D. W. Madden, "Simple and strong: Twisted silver painted nylon artificial muscle actuated by Joule heating," SPIE Smart Structures and Materials + Nondestructive Evaluation and Health Monitoring. International Society for Optics and Photonics (2014), 2014.

[4] A. J. Brizard, An introduction to Lagrangian mechanics, World Scientific, Singapore, 2008.

[5] K. K. Kar and A. K. Bhowmick, "High-strain hysteresis of rubber vulcanizates over a range of compositions, rates, and temperatures," Journal of Applied Polymer Science 67(5), pp. 1429-1439, 1997.

[6] R. Pelrine, R. Kornbluh, Q. Pei, and J. Jospeh, "High-speed electrically actuated elastomers with strain greater than 100\%," Science 287(5454), pp. 836-839, 2000.

[7] J. V. Humbeeck, "Non-medical applications of shape memory alloys.," Materials Science and Engineering: A 273, pp. 134-148, 1999. 